\title{
Characterization of the genetic switch from phage phi13 important for Staphylococcus aureus colonization in humans
}

\author{
Camilla Kristensen ${ }^{1}$, Anders Varming ${ }^{2}$, Helena Leinweber ${ }^{3}$, Karin Hammer ${ }^{1}$, Leila Lo \\ Leggio $^{2}$, Hanne Ingmer ${ }^{3}$, and Mogens Kilstrup ${ }^{1}$ \\ ${ }^{1}$ Technical University of Denmark \\ ${ }^{2}$ University of Copenhagen \\ ${ }^{3}$ University of Copenhagen Faculty of Life sciences
}

October 6, 2021

\begin{abstract}
Temperate phages are bacterial viruses that either reside integrated in a bacterial genome as lysogens or enter a lytic lifecycle. Decision between lifestyles is determined by a switch involving a phage-encoded repressor, CI, and a promoter region from which lytic and lysogenic genes are divergently transcribed. Here we investigate the switch of phage phi13 from the human pathogen Staphylococcus aureus. phi13 encodes several virulence factors and is prevalent in S. aureus strains colonizing humans. We show that the phi13 switch harbors a cI gene, a predicted mor (modulator of repression) gene, and three high-affinity operator sites binding CI. To quantify the decision between lytic and lysogenic lifestyle, we introduced reporter plasmids that carry the 1.3 $\mathrm{kb}$ switch region from phi13 with the lytic promoter fused to lacZ into S. aureus and B. subtilis. Analysis of beta-galactosidase expression indicated that decision frequency is independent of host factors. The white "lysogenic" phenotype, which relies on expression of cI, could be switched to a stable blue "lytic" phenotype by DNA damaging agents. We have characterized lifestyle decisions of phage phi13, and our approach may be applied to other temperate phages encoding virulence factors in S. aureus.
\end{abstract}

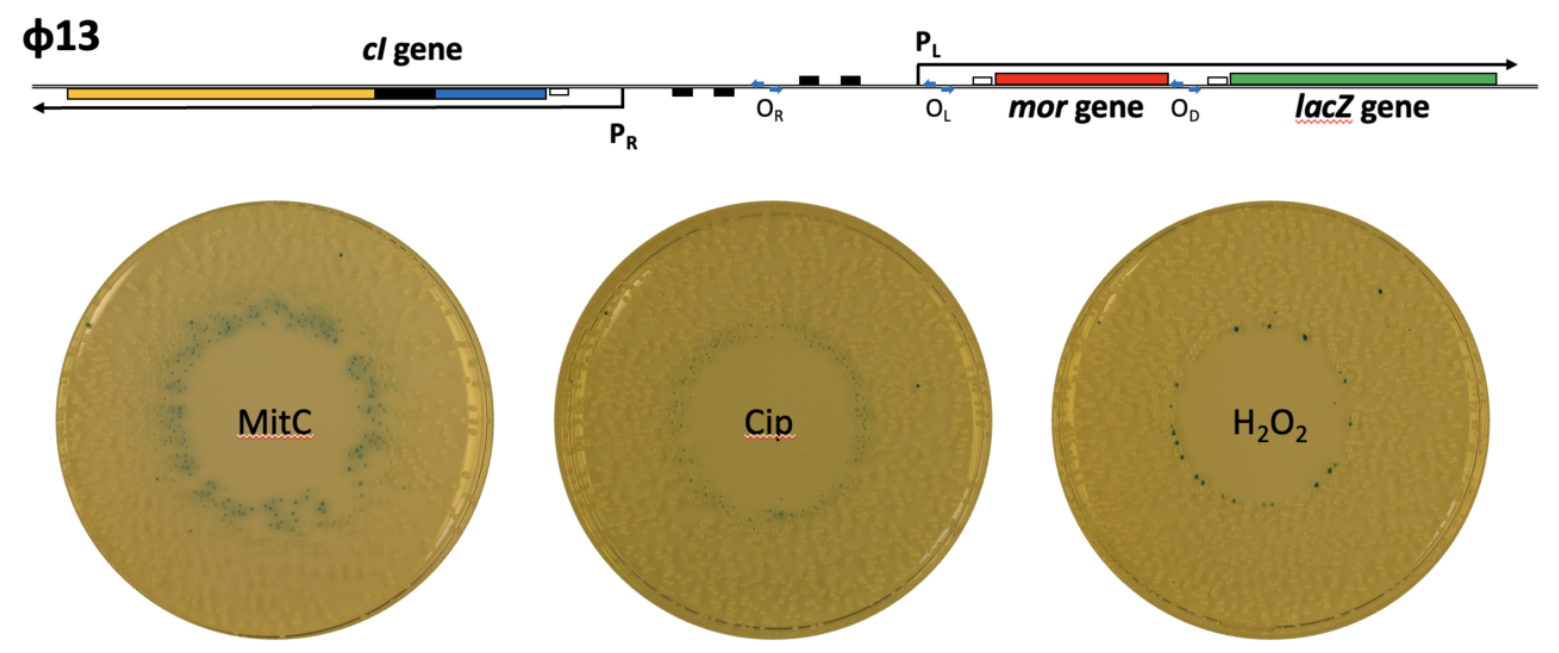




\section{Hosted file}

mbo32021070375.pdf available at https://authorea.com/users/439861/articles/540677characterization-of-the-genetic-switch-from-phage-phi13-important-for-staphylococcusaureus-colonization-in-humans 